\title{
1 Identification of rare loss of function variation regulating body fat distribution
}

2 Authors: Mine Koprulu ${ }^{\mathrm{a}}$, Yajie Zhao ${ }^{\mathrm{a} \#}$, Eleanor Wheeler ${ }^{\mathrm{a}}$, Liang Dong ${ }^{\mathrm{b}}$, Nuno Rocha ${ }^{\mathrm{b}}$, Satish Patel ${ }^{\mathrm{b}}$,

3 Marcel Van de Streek ${ }^{\mathrm{c}}$, Craig A. Glastonbury ${ }^{\mathrm{d}}$, Isobel D. Stewart a , Felix R. Daya, Jian'an Luan ${ }^{\mathrm{a}}$,

4 Nicholas Bowker $^{\mathrm{a}}$, Laura B. L. Wittemans ${ }^{\mathrm{a}}$, Nicola D. Kerrison ${ }^{\mathrm{a}}$, Debora M. E. Lucarelli ${ }^{\mathrm{a}}$, Inês Barroso ${ }^{\mathrm{e}}$,

5 Mark I. McCarthy ${ }^{\text {f }}$, Robert A. Scott ${ }^{\text {a }}$, Vladimir Saudek ${ }^{\text {b }}$, Kerrin S. Small ${ }^{\text {c }}$, Nicholas J. Wareham ${ }^{\text {a }}$,

6 Robert K. Semple ${ }^{\mathrm{g}}$, John R. B. Perry ${ }^{\mathrm{a}}$, Stephen O’Rahilly ${ }^{\mathrm{b}}$, Luca A. Lotta ${ }^{\mathrm{a}}$, Claudia Langenberg ${ }^{\mathrm{a}, \mathrm{h} *}$,

$7 \quad$ David B. Savage ${ }^{\mathrm{b} *}$

8 a. MRC Epidemiology Unit, University of Cambridge School of Clinical Medicine, Box 285 Institute of Metabolic Science, Cambridge Biomedical Campus, Cambridge, United Kingdom, CB2 0QQ

b. University of Cambridge Metabolic Research Laboratories, Wellcome Trust-MRC Institute of Metabolic Science, Box 289, Cambridge Biomedical Campus, Cambridge, United Kingdom, CB2 0 QQ

c. Department of Twin Research and Genetic Epidemiology, King's College London, St Thomas' Campus, Lambeth Palace Road, London, SE1 7EH, United Kingdom

d. BenevolentAI Limited, 4-8 Maple St, London W1T 5HD

e. Wellcome Sanger Institute, Hinxton, Cambridge, CB10 1SA, United Kingdom

f. Wellcome Centre for Human Genetics, University of Oxford, Roosevelt Drive, Oxford, OX3 7BN, United Kingdom

g. Centre for Cardiovascular Science, University of Edinburgh, 47 Little France Crescent, Edinburgh, EH16 4TJ, UK

* authors contributed equally

29 Claudia Langenberg (claudia.langenberg@mrc-epid.cam.ac.uk) 


\section{ABSTRACT}

33 Biological and translational insights from large-scale, array-based genetic studies of fat distribution, a key

34 determinant of metabolic health, have been limited by the difficulty in linking identified predominantly

35 non-coding variants to specific gene targets. Rare coding variant analyses provide greater confidence that

36 a specific gene is involved, but do not necessarily indicate whether gain or loss of function would be of

37 most therapeutic benefit. Here we use a dual approach that combines the power of genome-wide analysis

38 of array-based rare, non-synonymous variants in 184,246 individuals of UK Biobank with exome-sequence-

39 based rare loss of function gene burden testing. The data indicates that loss-of-function (LoF) of four genes

40 (PLINI, INSR, ACVRIC and PDE3B) is associated with a beneficial impact on $\mathrm{WHR}_{\mathrm{adjBMI}}$ and increased

41 gluteofemoral fat mass, whereas PLIN4 LoF adversely affects these parameters. This study robustly

42 implicates these genes in the regulation of fat distribution, providing new and in some cases somewhat

43 counter-intuitive insight into the potential consequences of targeting these molecules therapeutically.

45 Word count: 161 


\section{INTRODUCTION}

47 Fat distribution is a heritable trait, commonly estimated by the relative amounts of waist and hip fat (waistto-hip ratio, WHR) for a given body size. Genetic mechanisms linked to either relatively lower gluteofemoral or higher abdominal fat or both, have been shown to contribute to a greater WHR and its consistently adverse cardiometabolic consequences (1). Genome-wide array-based association studies have robustly identified many loci linked to WHR but thus far provided relatively limited biological and translational insights due to poor coverage of rare protein-coding variants and uncertainties connecting associated non-coding variants to functional genes $(2,3)$. Consequently, very few genes have been definitively linked to WHR and it is generally unknown whether a gain or loss of gene function is likely to drive observed associations.

The low frequency of rare (minor allele frequency $[\mathrm{MAF}]<0.5 \%$, as defined by the 1000 Genomes Project (4)) functional variants which may have sizable effects on the encoded protein, may be a consequence of selective pressure acting against them, and previous studies have shown inverse relationships between allele frequency and effect size for complex traits $(5,6)$. Rare variants that occurred recently are also likely to be in low linkage disequilibrium (LD) with nearby common variants, facilitating fine-mapping and identification of causal variants and genes (7). However, rare variants are difficult to impute (8) so their study requires large, homogeneous samples and direct genotyping. To date, the vast majority of studies have explored the contribution of common variants in relation to WHR including the largest meta-analysis of imputed genome-wide association studies which included up to 694,649 individuals but only identified two variants at MAF $0.1-0.5 \%$ (3). The only other study which investigated the role of rare variants for WHR was a subsequent trans-ethnic Exomechip effort that identified 9 low frequency or rare variants with a lowest MAF of $0.1 \%$ (9).

The contribution of the full spectrum of rare variants to WHR using sequence data has not been studied, yet has the potential to provide a more direct link between gene and phenotype, and to facilitate translation from gene identification to drug development. Whilst the identification of coding variants in a specific gene clearly increases confidence in linking that particular gene to a trait, the impact of individual coding variants can still be very, or at least relatively, subtle. Individual variant testing, even using exome sequencing data in large populations, therefore still provides limited power and leaves residual uncertainty about the benefits

74 of gain or loss of function of a particular gene. Exome wide scans of gene-based burden of rare loss-of-

75 function variants have the potential to address this limitation (10-14). In this study, we use a dual approach 76 that combines the power of large-scale genome-wide analysis of array-based rare, non-synonymous variants 
medRxiv preprint doi: https://doi.org/10.1101/2021.09.11.21263427; this version posted September 15, 2021. The copyright holder for this preprint (which was not certified by peer review) is the author/funder, who has granted medRxiv a license to display the preprint in perpetuity.

It is made available under a CC-BY-NC-ND 4.0 International license .

78 pathways regulating body shape and fat distribution (assessed by BMI-adjusted WHR [WHR $\mathrm{WdjBMI}_{\text {) }}$ ) and to

79 determine their effects on body composition and metabolic health.

\section{RESULTS}

81 A genome-wide analysis of directly genotyped, rare $(0.1 \% \leq \mathrm{MAF} \leq 0.5 \%)$ non-synonymous variants 82 associated with $\mathrm{WHR}_{\text {adjBMI }}$ at $p<5 \times 10^{-8}$ in 450,562 European ancestry individuals from UK Biobank 83 identified lead variants in PLIN1 p.L90P (rs139271800, EAF=0.1\%), PDE3B p.R783X (rs150090666, $84 \mathrm{EAF}=0.1 \%$ ), ACVRIC p.I195T (rs56188432, EAF=0.2\%), CALCRL p.L87P (rs61739909; EAF=0.3\%), 85 ABHD15 p.G147D (rs141385558; EAF=0.2\%) and PYGM p.R50X (rs116987552, EAF=0.4\%) (Figure 1,

86 Supplementary Table 1). We observed a correlation of 0.99 and minor allele concordance of 0.99 comparing 87 genotyped to whole-exome sequenced rare $(0.1 \leq \mathrm{MAF} \leq 0.5 \%)$ non-synonymous variants when testing the 88 validity of rare, genotyped variants using exome-sequencing data from the overlapping samples 89 (Supplementary Table 2).

\section{Sex-differences in genetic effects of rare variants on $W H R_{\text {adjBMI }}$}

91 Common variant analyses have provided evidence of differences in genetic associations with fat distribution 92 between men and women (3). In line with this, we found evidence of significant sex interactions, with 93 stronger genetic effects in women, compared to men for all lead variants, except for ACVRIC p.I195T and 94 PYGM p.R50X (Table 1). We therefore conducted sex-specific analyses which revealed two additional 95 variants, $P L C B 3$ p.V806I (rs145502455, EAF=0.4\%) and FNIP1 p.R518Q (rs115209326, EAF=0.3\%) to 96 be genome-significant in $\left(p<5 \times 10^{-8}\right)$ in women, with no effect in men (Table 1, Supplementary Table 1). 97 No variants reached genome-wide significance in men only. 
medRxiv preprint doi: https://doi.org/10.1101/2021.09.11.21263427; this version posted September 15, 2021. The copyright holder for this preprint (which was not certified by peer review) is the author/funder, who has granted medRxiv a license to display the preprint in perpetuity.

It is made available under a CC-BY-NC-ND 4.0 International license .
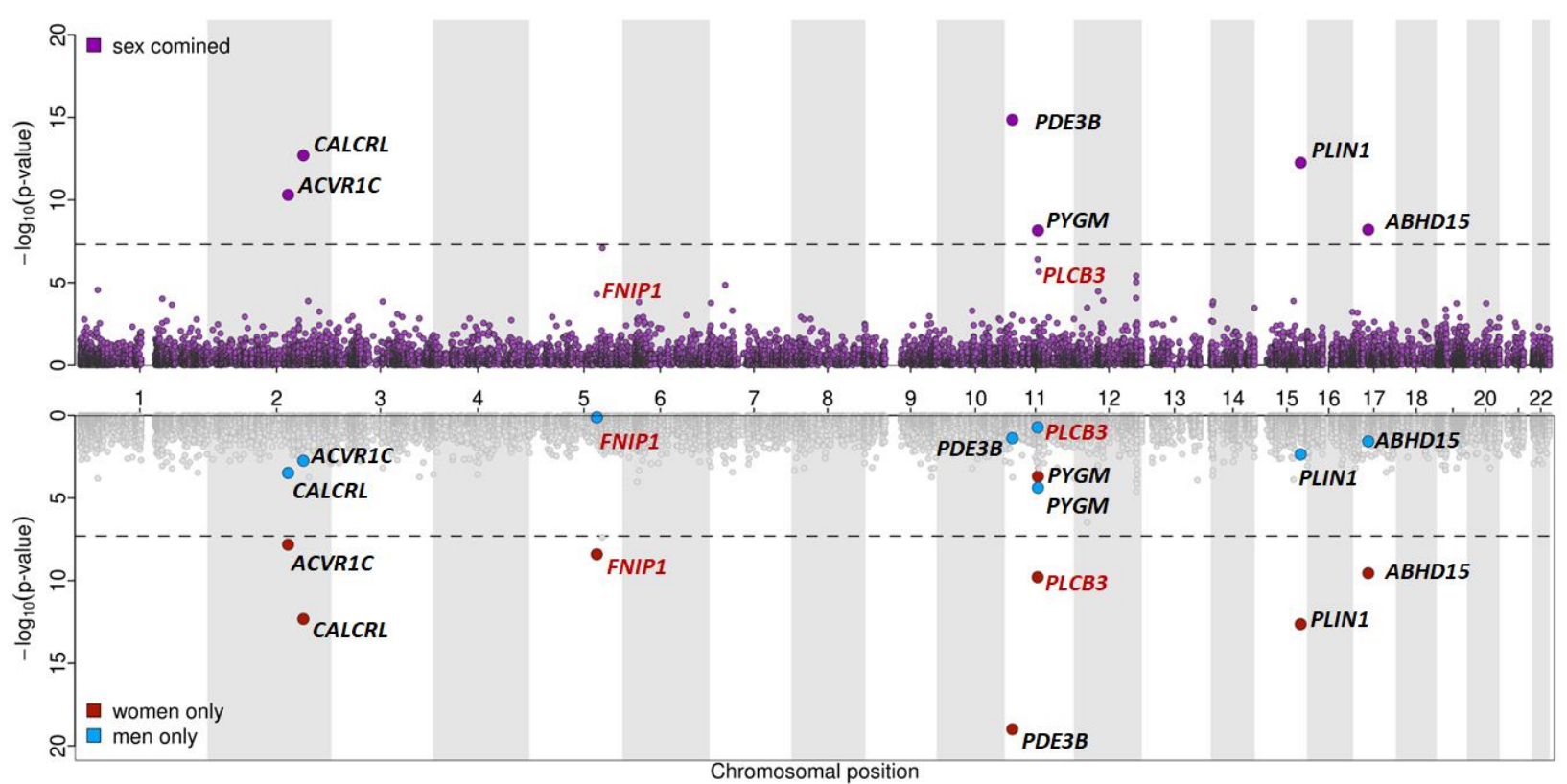

Figure 1. Miami plot for sex-combined and sex-specific single marker association results for WHRadjBMI. Top: Manhattan plot representing results from the main, sex-combined GWAS for WHR adjBMI for genotyped, rare nonsynonymous variants $(0.1 \% \leq \mathrm{MAF} \leq 0.5 \%$, correlation and rare allele concordance $>0.9$ when compared to the exome sequencing data). Gene annotations for the genome-wide significant variants from the main, sex-combined analyses are shown in black; gene annotations and significance from the main, sex-combined analyses for variants that were genome-wide significant in sex-specific analyses (women) only are shown in red. Bottom: Sex-specific significance of the variants highlighted above.

\begin{tabular}{ccccccccc}
\hline Variant & rsID & $\begin{array}{c}\text { Sex } \\
\text { interaction } \\
\boldsymbol{p} \text { value }\end{array}$ & $\begin{array}{c}\boldsymbol{p} \text { value } \\
\text { Women }\end{array}$ & $\begin{array}{c}\boldsymbol{p} \text { value } \\
\text { Men }\end{array}$ & $\begin{array}{c}\text { Beta } \\
\text { Women }\end{array}$ & $\begin{array}{c}\text { Beta } \\
\text { Men }\end{array}$ & $\begin{array}{c}\text { SE } \\
\text { Women }\end{array}$ & $\begin{array}{c}\text { SE } \\
\text { Men }\end{array}$ \\
\hline PLIN1 p.L90P & rs139271800 & $4.07 \times 10^{-3}$ & $1.90 \times 10^{-13}$ & $4.50 \times 10^{-3}$ & -0.273 & -0.126 & 0.039 & 0.042 \\
PDE3B p.R783X & rs150090666 & $5.66 \times 10^{-5}$ & $1.00 \times 10^{-19}$ & $4.10 \times 10^{-2}$ & -0.392 & -0.102 & 0.042 & 0.048 \\
ACVR1C p.I195T & rs56188432 & $3.61 \times 10^{-1}$ & $1.10 \times 10^{-8}$ & $3.40 \times 10^{-4}$ & -0.16 & -0.109 & 0.028 & 0.032 \\
CALCRL p.L87P & rs61739909 & $4.30 \times 10^{-3}$ & $4.30 \times 10^{-13}$ & $2.50 \times 10^{-3}$ & -0.171 & -0.082 & 0.024 & 0.026 \\
ABHD15 p.G147D & $\mathrm{rs} 141385558$ & $4.53 \times 10^{-3}$ & $3.40 \times 10^{-10}$ & $3.80 \times 10^{-2}$ & 0.168 & 0.057 & 0.026 & 0.029 \\
PYGM p.R50X & $\mathrm{rs} 116987552$ & $3.56 \times 10^{-1}$ & $2.90 \times 10^{-4}$ & $5.40 \times 10^{-5}$ & 0.079 & 0.095 & 0.021 & 0.024 \\
PLCB3 p.V806I & $\mathrm{rs} 145502455$ & $2.34 \times 10^{-2}$ & $1.60 \times 10^{-10}$ & $2.00 \times 10^{-1}$ & 0.126 & 0.029 & 0.021 & 0.024 \\
FNIP1 p.R518Q & $\mathrm{rs} 115209326$ & $6.09 \times 10^{-4}$ & $4.80 \times 10^{-9}$ & $8.40 \times 10^{-1}$ & -0.128 & -0.003 & 0.023 & 0.026 \\
\hline
\end{tabular}

Table 1: Sex-stratified results for variants identified in joint and sex specific analyses of genotyped rare variants 
110

BOLT LMM p-value in women; $p$ value Men, BOLT LMM p-value in men; Beta Women, effect size in women; Beta Men, effect size in men; SE Women, standard error in women; SE Men, standard error in men.

\section{Genomic context and fine-mapping analyses}

We found strong statistical evidence for causal associations of rare non-synonymous variants in PLINI, PDE3B, ACVRIC and CALCRL (Supplementary Note 1, Supplementary Table 3, Supplementary Figure 1) through conditional analysis and fine-mapping, whereas genomic context analyses did not support the causality of the identified rare lead variants in ABHD15 or PYGM from the joint (sex-combined) analysis, and of PLCB3 and FNIP1 in the women-only analysis (Supplementary Note 2, Supplementary Table 4). Bioinformatic analysis of these variants strongly predicted that the $P D E 3 B$ variant $\mathrm{p} . \mathrm{R} 783 \mathrm{X}$ would truncate $P D E 3 B$ within the catalytic site, impairing $P D E 3 B$ catalytic activity if expressed (Supplementary Note 3), whereas predictions of the functional impact of the PLIN1, ACVRIC and CALCRL variants were less conclusive (Supplementary Note 3).

\section{Exome-sequenced based burden testing of rare, loss-of function variants}

Next, we considered the genes identified in the single variant analysis for exome-sequence-based gene rare LoF and missense burden testing in 184,246 individuals in UK Biobank (see Methods, Gene-based association testing) and found that PLIN1, PDE3B, ACVRIC, and CALCRL were all significantly associated with lower $\mathrm{WHR}_{\mathrm{adjBMI}}$ at a Bonferroni corrected threshold $(p<0.0125)$. Predicted loss of function (pLoF) variants showed the most significant association for PLINI and PDE3B, moderate impact variants for $C A L C R L$, and the combination of $\mathrm{pLoF}$ with moderate impact variants for ACVRIC (Supplementary Table $5)$.

In order to identify additional genes where loss of function may regulate fat distribution, we extended this approach to a hypothesis free, exome-wide analysis $\left(p<2.53 \times 10^{-6}\right)$ for $\mathrm{WHR}_{\text {adjBMI }}$ using more stringent quality control (QC) parameters (see Methods). This identified PLIN4 and INSR in at least one of the variant categories (see Methods), in addition to PLIN1, ACVRIC and PDE3B (Figure 2, Supplementary Table 6). PLIN4, INSR and PDE3B all showed significantly larger standardized effect sizes for women compared to men $(p<0.05)$ in gene-based analyses, in line with the single marker results (Supplementary Table 7). 


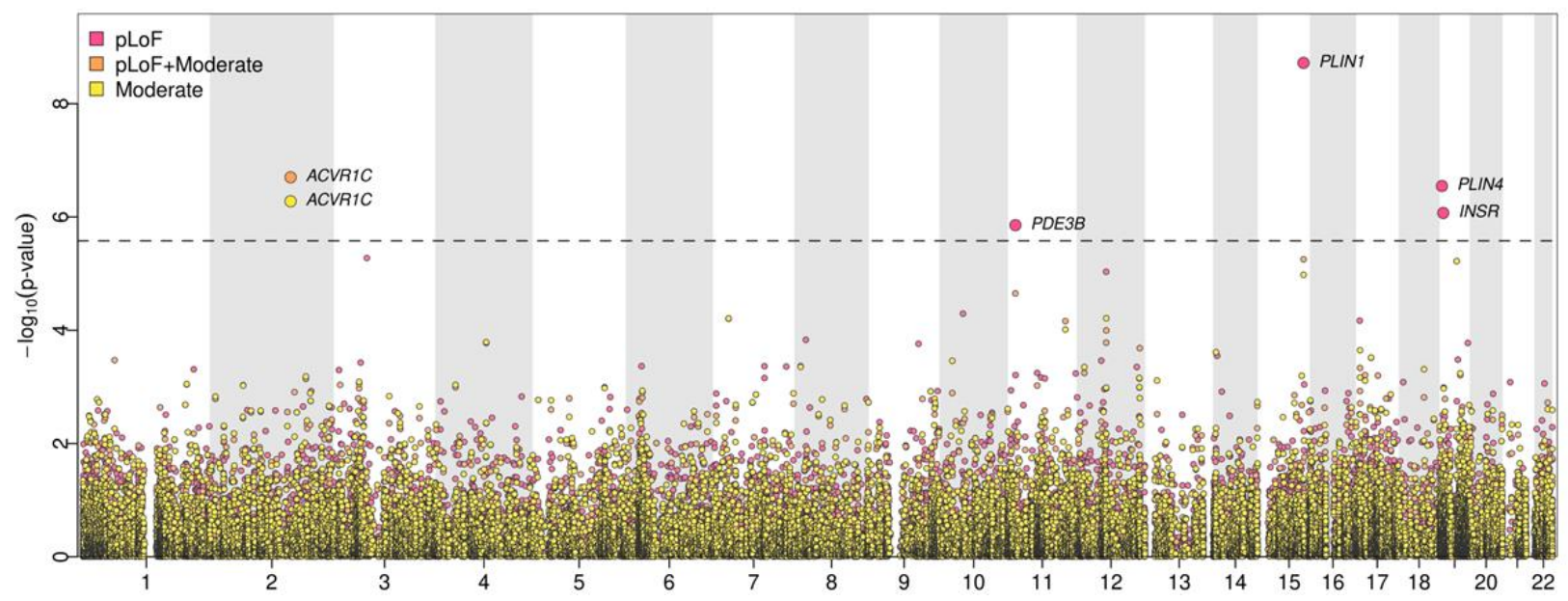

Figure 2. Gene-based association results. Gene-based exome-wide discovery results for $\mathrm{WHR}_{\text {adjBMI. }}$ The horizontal dashed line represents the exome-wide significance threshold $\left(p=2.53 \times 10^{-6}\right)$.

While the joint effect of rare LoF variants in PLIN4 (65 variants, 1,065 carriers) was associated with a higher $\mathrm{WHR}_{\text {adjBMI }}\left(\right.$ Beta $\left.=0.16[0.10-0.22], p=5.86 \times 10^{-7}\right)$, the combination of rare LoF variants in PLIN1 (31 variants, 393 carriers) was associated with a lower WHR $_{\text {adjBMI, }}\left(\right.$ Beta $=-0.27$ [-0.17 - -0.36], $p=9.82 \times 10^{-}$ 9) (Supplementary Table 6). The lead PLIN1 LoF variant (PLIN1 p.T338DfsX51, rs750619494) is predicted to result in a frameshift from amino acid 338 with a premature stop at amino acid 388, though it may well be subject to nonsense mediated RNA decay. Several additional PLINI variants are similarly expected to result in early truncations or nonsense mediated RNA decay (Supplementary Table 8). In either instance, these variants are expected to impair Plin1 interaction with $A B H D 5$ and thus it's regulation of adipose triglyceride lipase (ATGL) (15). In the case of PLIN4 (p.Q372X, rs201581703), the variant list also included early frameshift/premature stop variants predicted to result in nonsense mediated RNA decay.

We next assessed phenotypic associations with refined measures of fat distribution and cardiometabolic parameters and diseases. Bioelectrical Impedance Analysis (BIA) derived body fat compartment measurements (16) showed that PLIN4 (pLoF) was associated with higher android and trunk fat, and lower gynoid and leg fat (Figure 3, Supplementary Figure 2, Supplementary Table 9) whereas PLIN1 (pLoF) acted in the opposite direction. Fat distribution is strongly linked to insulin resistance, but as direct indicators of insulin resistance are not currently available in UK Biobank, we evaluated the impact of these genes on metabolic indicators typically associated with insulin resistance $(17,18)$ (Figure 3, Supplementary Figure 2, Supplementary Table 9). PLIN4 LoF was associated with higher triglycerides (TGs), TG/HDL (triglyceride/high-density lipoprotein cholesterol) ratio and higher HbA1c levels. The associations for PLIN1 consistently contrasted with those of PLIN4 with lower TGs, TG/HDL ratio and additionally higher 
HDL cholesterol levels, in keeping with a beneficial impact on insulin sensitivity. In keeping with these findings, PLIN4 LoF was nominally associated with an increased risk for type 2 diabetes (T2D) (OR=1.36 [1.06-1.66], $p=0.04)$ in the Type 2 Diabetes Knowledge Portal (T2DKP; https://t2d.hugeamp.org/), though none of the genes showed a significant association with T2D in UK Biobank through our analysis or in the AstraZeneca PheWAS Portal (https://azphewas.com/) (Supplementary Figure 2; Supplementary Table 9; Supplementary Table 10). PLIN1 LoF showed nominal significance for a lower risk of cardiovascular heart disease $(\mathrm{CHD})(p=0.03, \mathrm{OR}=0.55[0.31-0.91])$ in our analysis of UK Biobank, a finding supported by [0.32-0.75], $\left.p=4.49 \times 10^{-4}\right)$ in the AstraZeneca PheWAS Portal (14).

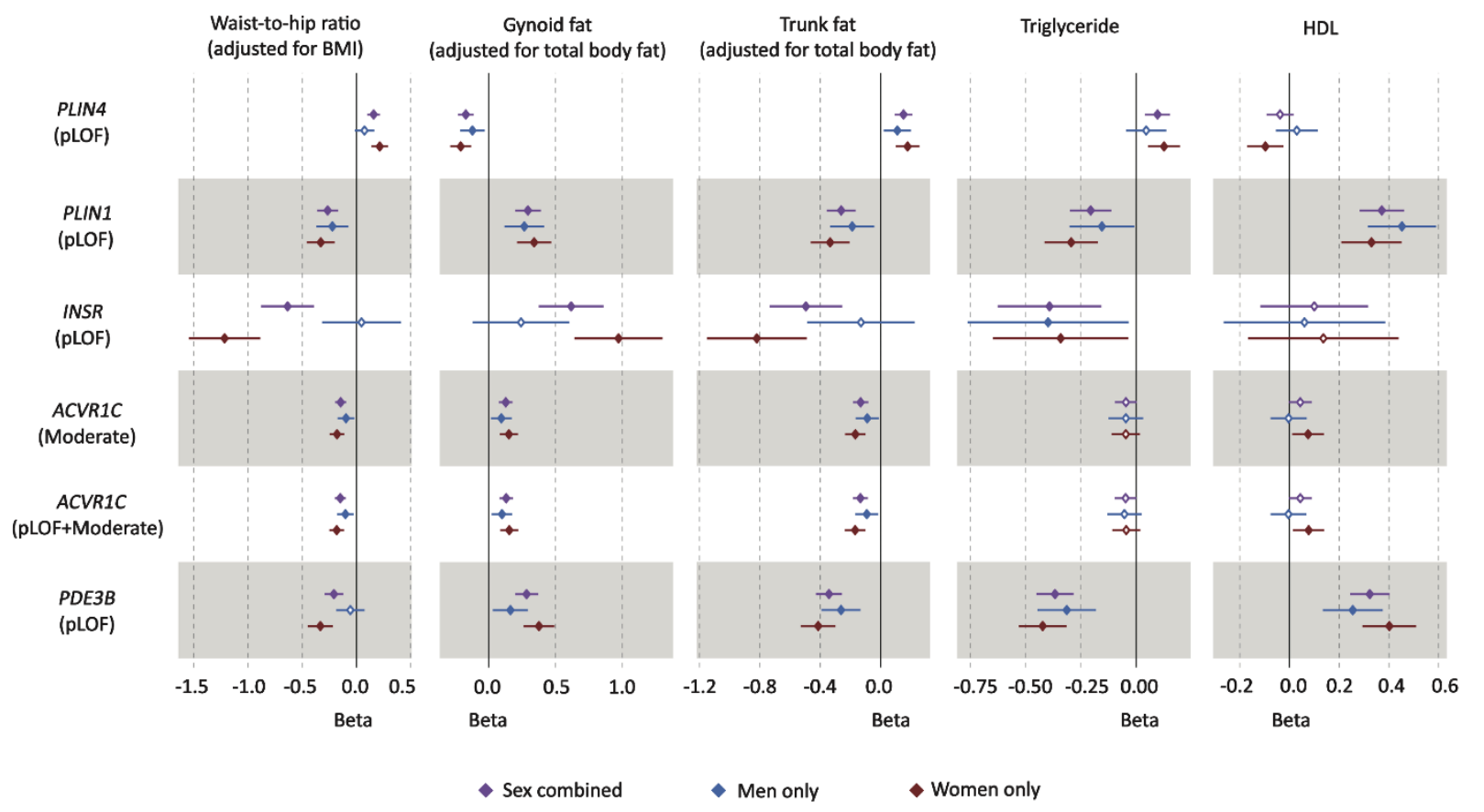

Figure 3. Forest plot of phenotypic associations for significant variant-gene categories. Black represents the sexcombined, blue represents the men-only and red represents the women-only analysis. Horizontal lines represent 95\% confidence intervals. Waist-to-hip ratio adjusted for BMI $(\mathrm{n}=184,246)$, gynoid fat adjusted for total body fat $(\mathrm{n}=$ $178,143)$, trunk fat adjusted for total body fat $(178,143)$, triglyceride levels $(\mathrm{n}=175,271)$ and HDL cholesterol $(\mathrm{n}=161,239)$ were all driven from UK Biobank (See Supplementary Table 12 for details).

175 Similarly to PLIN1, the combined effect of LoF variants in the INSR (27 variants, 61 carriers) was

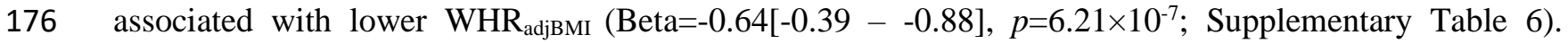
177 Although a few common intronic variants (rs1035942, rs1035940, rs62124511, rs34194998) and a low- 
frequency synonymous variant (rs1799815) in the INSR have previously been associated with WHR $_{\text {adjBMI }}$ $(3,19)$, the causal mechanism underlying these associations remains unknown. Our gene-based findings indicate that the INSR can alter body fat distribution through loss of function. Given the fact that the INSR gene encodes the insulin receptor itself and that both biallelic and heterozygous loss of function variants in this gene have long been linked with monogenic severe insulin resistance syndromes (20), this evidence for loss of function of the INSR having a seemingly beneficial effect on fat distribution i.e. lower $\mathrm{WHR}_{\text {adjBMI }}$ is surprising. Importantly, none of the INSR mutations previously linked to monogenic disease were present in our UK Biobank analysis. The lead INSR variant (p.R525X) is predicted to result in truncation of the protein within the extracellular domain preventing interaction of the extra- and intra-cellular domains, and thus formation of a functional receptor. In the homozygous state, this would be expected to lead to monogenic severe insulin resistance. In terms of body fat distribution, heterozygous INSR LoF was also associated with higher gynoid and leg fat, and lower android and trunk fat mass (Figure 3, Supplementary Figure 2, Supplementary Table 9). Similarly to the cardio-metabolic associations of PLIN1 indicating a beneficial effect, heterozygous loss of INSR was associated with lower TGs and a lower TG/HDL ratio (Figure 3, Supplementary Figure 2, Supplementary Table 9). It was also associated with lower LDL (lowdensity lipoprotein) cholesterol levels but was not associated with altered HDL (Figure 3, Supplementary Figure 2, Supplementary Table 9). Despite these seemingly favourable changes in fat distribution and plasma lipids, INSR LoF showed a nominal association for increased susceptibility to T2D in the T2DKP $(\mathrm{OR}=3.67[2.50-4.83], p=0.02)$ (Supplementary Table 10).

For $A C V R I C$, the genetic architecture of gene-based results was slightly different, gene-based association results were significant for (i) the combined burden of $\mathrm{pLoF}$ and moderate impact variants and (ii) for moderate impact variants only. There were 130 rare moderate impact variants and 9 rare high impact variants included in this analysis [1414 and 16 carriers, respectively]. The combined effect of pLoF and moderate impact variants and moderate impact only variants were both associated with lower $\mathrm{WHR}_{\text {adjBMI }}$ (Beta $=-0.15[-0.10--0.20]$ and $-0.15[-0.10--0.20], p=1.68 \times 10^{-7}$ and $4.57 \times 10^{-7}$, respectively; Supplementary Table 6). In this instance, the highest-ranking variant was the previously reported p.I195T variant $(19,21)$. In silico predictions including M-CAP (22), REVEL (23), SIFT (24), PolyPhen-2 (25) and PROVEAN (26) all asses this variant to be damaging to the protein and structural modelling also suggests that it is likely to have a sizable impact (Supplementary Note 3). CADD (27) also estimates this variant to be among the top $1 \%$ of the deleterious variants ranked by CADD (score $=27.1$ ). To test this prediction, we performed a luciferase reporter assay in HEK293 cells which strongly suggested that the mutation impairs $A C V R I C$ signalling (Figure 4). 


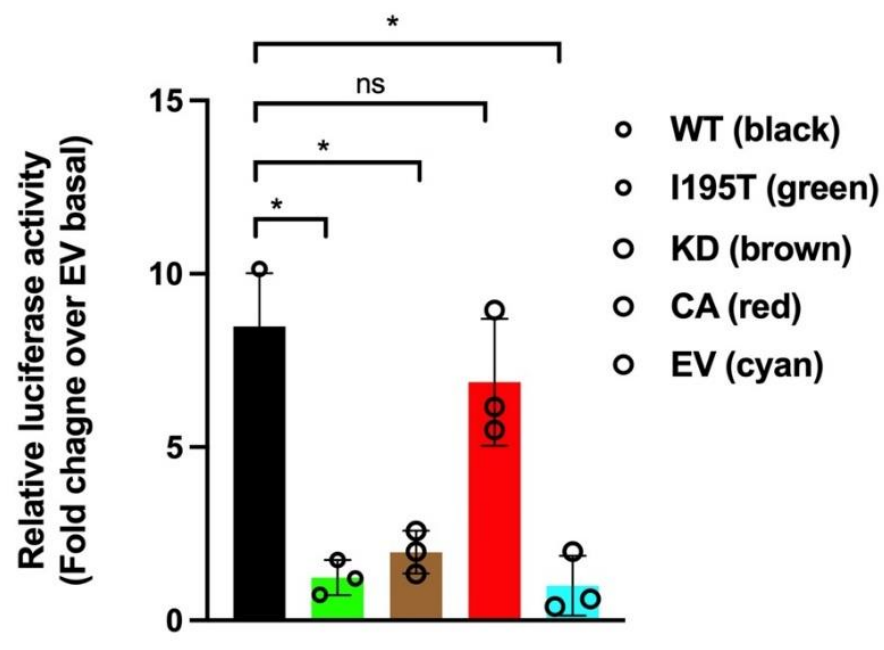

Figure 4. Functional impact of ACVR1C I195T variant on Smad signalling. HEK293 cells were transiently transfected with ACVR1C expression constructs and their receptor components, along with firefly and Renilla luciferase expression plasmids. Firefly luciferase activity was normalised to Renilla activity and the luciferase activity in non-stimulated cells transfected with empty vector (EV) was set to 1 . A constitutively active (CA) ACVR1C variant T194D and a kinase dead (KD) variant K222R were included for comparison. Results from three independent experiments are presented as mean \pm SD. Statistical significance was evaluated by one-way ANOVA with Tukey's post hoc test for multiple comparisons between pairs. WT, wild type ACVR1C. * $\mathbf{P}<$ 0.001 , ns not significant.

The phenotypic associations for ACVRIC LoF for fat categories were similar to PLIN1 LoF, with significant associations with higher gynoid and leg fat, and lower android and trunk fat. However, the cardio-metabolic associations were less clear for ACVRIC with an association with lower TG and but not HDL or the TG/HDL ratio (Figure 3, Supplementary Figure 2; Supplementary Table 9).

Finally, the combined effect of LoF variants in $P D E 3 B$ was also significantly associated with lower

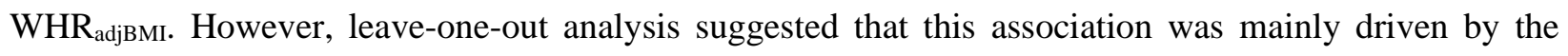
premature stop variant (p.R783X, rs150090666; $p$-value after dropping the variant=0.49; Supplementary Table 11). All other candidate genes remained at least nominally significant after dropping the most significant variant (Supplementary Table 11). Our analysis of the PDE3B p.R783X variant was in line with previous reports associating it with lower triglyceride levels and higher HDL $(28,29)$. PDE3B p.R783X has also been reported to be associated with higher apolipoprotein B, lower apolipoprotein A1 levels and other haematological traits (30). This variant was reported to be significantly associated with cardiovascular disease when meta-analysed in UK Biobank and other cohorts $(29,31)$. 


\section{DISCUSSION}

239 Central adiposity has long been linked to insulin resistance and metabolic disease (32-37) but exactly why this is the case and, other than sex hormones, what exactly determines fat distribution remains incompletely understood. So, what have we learnt from human genetics thus far? Firstly, that inheritance contributes to WHR $(3,38)$. Secondly, monogenic partial lipodystrophies indicate that single gene variants can be sufficient to mediate substantial changes in fat distribution, classic examples being mutations in $L M N A$ and PPARG. Interestingly, both proteins are expressed in all white adipocytes and yet specific loss of function variants are consistently associated with loss of hip and leg fat whereas visceral fat is preserved (39).

Thirdly, whilst the beneficial impact of thiazolidinediones, one of very few drugs that clearly improve insulin sensitivity, was recognised before the discovery of PPARG mutations in patients with partial lipodystrophy, this link attests to the potential for human genetics to inform drug discovery. Fourthly, GWAS studies have identified many loci associated with $\operatorname{WHR}(2,3,9)$ though these have yet to be translated into therapeutic targets. Finally, Mendelian Randomization has been used to establish that genetic mechanisms linked to greater $\mathrm{WHR}_{\text {adjBMI }}$ can be causally linked to the risk of cardiovascular disease and type 2 diabetes through either relatively lower gluteofemoral or higher abdominal fat or both (40). Furthermore, these associations are very likely to be underpinned by insulin resistance as the genetic risk score for $\mathrm{WHR}_{\text {adjBMI }}$ was also shown to be strongly associated with elevated fasting insulin, higher triglycerides and lower HDL cholesterol (40).

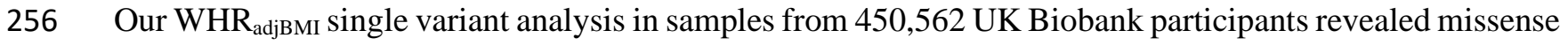

257 variants in 3 genes (CALCRL, PLIN1 and ACVRIC) and a nonsense variant in PDE3B. All these genes are

258 highly expressed in adipose tissue in keeping with emerging evidence that adiposity itself is largely centrally mediated whereas where excess energy is stored is regulated within adipose tissue itself $(2,41)$. $C A L C R L$ is also expressed in a host of other tissues and its role in adipose tissue remains to be established $(42,43)$. PLIN1 is a lipid droplet surface protein almost exclusively expressed in adipocytes and has a wellestablished role in regulating both triglyceride and diacylglycerol hydrolysis (44). PDE3B is expressed in many tissues but has long been linked to adipocyte lipolysis, and specifically to insulin mediated inhibition of lipolysis $(45,46)$. Several lines of evidence have recently implicated ACVRIC in the regulation of lipolysis, but it is expressed in many tissues in addition to adipose tissue and further work is required to convincingly establish exactly what it does in adipocytes (47-49).

In terms of the impact of the specific mutations present in each gene, the PDE3B p.R783X is clearly expected to impair $P D E 3 B$ catalytic activity, and thus potentially to increase cAMP levels and lipolysis, but the impact of the other three variants is far less certain (see Supplementary Note 3). Interestingly, gene based LoF burden analyses for all four genes were at least nominally significant, suggesting that the single 
variants were most likely to impair function of the encoded proteins. At least when transiently transfected

272 into cultured cells, our functional data is also consistent with the LoF predictions for the ACVRIC p.I195T

273 variant.

274 Our exome wide analyses confirmed significant effects of LoF variants in PLIN1, ACVRIC and PDE3B. In

275

276

277

278

279

280

281

282

283 the cases of $A C V R I C$ and $P D E 3 B$, the lead $\mathrm{LoF}$ variants were the same as single variants reported above, namely the ACVRIC p.I195T and PDE3B p.R783X variants. The fact that the phenotypic associations of the PLIN1 p.L90P variant are directionally consistent with the PLIN1 LoF gene burden data suggests that this variant is likely to be a loss of function variant too. Both the lead PLINI variant and several additional PLIN1 variants are expected to result in early truncations or nonsense mediated RNA decay. These data are consistent with Laver et al.'s assertion that PLIN1 haploinsufficiency is not associated with lipodystrophy (50). Interestingly, several heterozygous PLIN1 frameshift variants had previously been linked to partial lipodystrophy (51). None of these variants overlap with those identified in UK Biobank to date and none are predicted to result in nonsense mediated RNA decay. Instead, in several cases, immunoblotting of adipose tissue lysates confirmed expression of an elongated form of Perilipin 1 in addition to the wildtype copy, so perhaps expression of these mutant forms with an altered carboxy-terminus, accounts for the seemingly 'opposite' phenotypes $(51,52)$.

Gene burden testing also highlighted the role of PLIN4 LoF variants in fat distribution, though in this case,

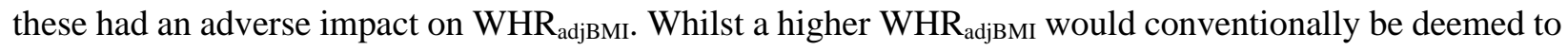
be metabolically adverse, it is possible that this need not be the case for all genetic perturbations, however, our phenotypic analyses were consistent with the predicted outcomes for all the above genes, in fact, the phenotypic associations for PLIN1 and PLIN4 were consistently opposite. Similar to PLIN1, PLIN4 is highly expressed in adipose tissue, but it is also expressed in heart and skeletal muscle, and the PLIN4 knockout mouse has not been reported to have an adipose tissue phenotype to date (53).

The last gene identified in the exome wide gene burden analysis was the INSR. In this instance, the lead variant (p.R525X) is expected to truncate the protein in the alpha subunit shortly before the disulphide bond normally connecting alpha and beta subunits. This is expected to abrogate synthesis of functional receptor. Even if truncated protein were synthesised this would not be able to dimerise with or exert dominant negative activity over co-expressed wild type receptor, and so heterozygosity for the truncating variant would be expected to reduce functional receptor protein by $\sim 50 \%$. In keeping with this, biallelic mutations in this domain usually cause extreme IR classified as Donohue or Rabson-Mendenhall syndrome. Parents of affected children have not been systematically studied and are generally held to be metabolically normal. In contrast, heterozygous INSR variants in the intracellular beta subunit, which are synthesised and interfere 
with wild type receptor function, cause type A insulin resistance (20). Whilst fat mass is often reduced in Donohue's syndrome, heterozygous variants associated with type A insulin resistance are not reported to be associated with fat redistribution and interestingly do not typically lead to fatty liver or dyslipidaemia (20). Our data suggest, surprisingly, that the INSR LoF variants favourably impact WHR adjBMI and LDL cholesterol. Whilst the INSR LoF association with T2D was relatively weak statistically and not seen in all the cohorts assessed, it is conceivable that INSR LoF might adversely affect pancreatic beta cell function and/or insulin sensitivity despite the apparently beneficial impact on fat distribution. The change in triglycerides is somewhat reminiscent of the well described absence of dyslipidaemia in patients with monogenic severe insulin resistance due to bi- or mono-allelic INSR mutations, so again this does not preclude the INSR LOF variants being associated with reduced insulin sensitivity.

Our sex-specific analyses consistently revealed stronger effects in women than in men. These data are consistent with the fact that WHR is more strongly associated with insulin resistance in women than in men (54). Fat mass in women is consistently significantly higher than men of a similar BMI, who typically have higher lean/muscle mass. The adverse impact of a lack of lower limb/gluteofemoral fat on metabolism is strikingly apparent in patients with familial partial lipodystrophy, particularly types 2 and 3, due to specific mutations in LMNA and PPARG respectively (39). In these and in fact in all forms of partial lipodystrophy, metabolic disease manifests considerably earlier and is typically more severe in women than in men (39, $55,56)$.

Our analyses have several limitations which future work should help to resolve. Firstly, the statistical power to detect associations, particularly when examining rare variants, depends on the sample size. Hence, there is the opportunity to discover additional findings when the WES data is released in the full UK Biobank cohort or other large-scale studies. Secondly, the phenotypic follow up of cardiometabolic diseases for the candidate genes was primarily conducted in UK Biobank, a population cohort with a limited number of cases of specific diseases. Our follow-up in T2DKP revealed the potential of datasets enriched for cases to increase statistical power in phenotypic follow ups. Thirdly, fat distribution is strongly associated with insulin resistance, but the UK Biobank cohort did not provide fasting samples so direct measures of insulin and inferred indices of insulin sensitivity are not available.

In conclusion, our analysis strongly implicates at least four genes in the regulation of fat distribution. Furthermore, the data suggests that inhibitors of PLIN1, PDE3B and ACVRIC might favourably impact fat distribution and associated metabolic phenotypes whereas PLIN4 inhibition is likely to have adverse health consequences. The data in PLIN1 needs to be tempered by the earlier reports linking some specific PLINI LoF variants with partial lipodystrophy. Finally, the data on the INSR seemed to suggest a potential disconnect between an apparently favourable impact of LoF variants on WHR and an apparently adverse 
medRxiv preprint doi: https://doi.org/10.1101/2021.09.11.21263427; this version posted September 15, 2021. The copyright holder for this preprint (which was not certified by peer review) is the author/funder, who has granted medRxiv a license to display the preprint in perpetuity.

It is made available under a CC-BY-NC-ND 4.0 International license .

336 impact on T2D risk. These findings provide valuable insight into the potential of these genes as therapeutic 337 targets.

338 


\section{METHODS}

\section{The UK Biobank Resource}

The UK Biobank is a large-scale prospective population-based study of approximately 500,000 participants aged $40-69$ at the time of recruitment (57). Recruitment took place between $2006-2010$ in centres across the United Kingdom and participants have deep phenotypic information collected from initial and repeat assessment visits, health records, self-reported survey information, linkage to death and cancer registries, urine and blood biomarkers and other phenotypic endpoints. A Seca $200 \mathrm{~cm}$ tape was used to measure waist and hip circumference at the baseline visit, and BMI was calculated from height and weight measurements. WHR adjBMI was constructed as the ratio of waist and hip circumferences adjusted for age, age $^{2}$ and BMI (measured at the baseline assessment visit). Residuals were calculated for men and women separately and then transformed using the rank-based inverse normal transformation. All additional phenotypes are described in Supplementary Table 12.

\section{Genome-wide association scan of genotyped rare nonsynonymous genetic variants}

354 Genetic variants were genotyped in UK Biobank using the Affymetrix UK BiLEVE or the Affymetrix UK Biobank Axiom arrays (57). Genotyping underwent quality control procedures including (a) routine quality checks carried out during the process of sample retrieval, DNA extraction, and genotype calling; (b) checks and filters for genotype batch effects, plate effects, departures from Hardy Weinberg equilibrium, sex effects, array effects, and discordance across control replicates; and (c) individual and genetic variant call rate filters as previously described (57). We further excluded genetic variants with a genotype call rate below $95 \%$ and variants that were (i) not rare $(0.1 \% \leq \mathrm{MAF} \leq 0.5 \%)$ or (ii) not non-synonymous or (iii) had poor correlation $(\mathrm{r}<0.9)$ or rare allele concordance $(<0.9)$ when compared to the whole exome sequence data (Supplementary Table 2). Genomic annotations were performed using the ANNOVAR software (58). The coordinates of genotyped rare variants were lifted over from GRCh37 to GRCh38 using liftOver and all reported positions in this study are in GRCh38. A total of 13,181 genetic variants in 7,481 genes were available for analysis. Genome-wide association analyses were performed using the BOLT-LMM software (59) in 450,562 participants of European Ancestry defined using a K-means clustering approach applied to the first four principal components calculated from genome-wide SNP genotypes. the variants identified in the sex combined analysis were formally tested in unrelated individuals using a 
371 linear regression model including an interaction term between the genetic variant and sex using the same

372 covariates used in the discovery analysis.

373

\section{Conditional analyses and fine-mapping}

375 At each associated genomic region, we conducted systematic analyses of the genomic context of 376 associations. Our goal was to establish whether or not the identified rare nonsynonymous variants are likely

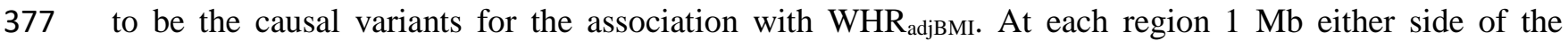
378 nonsynonymous genetic variants associated with WHR $_{\text {adjBMI, }}$ we conducted both approximate and formal 379 conditional analyses. We considered the association of all genetic variants in the regions regardless of 380 functional annotation or allele frequency using directly-genotyped and imputed data (imputed using the 381 Haplotype Reference Consortium (HRC) and UK10K haplotype resource). First, approximate conditional analyses were conducted on summary-level estimates using GCTA $(60,61)$ to identify sets of conditionallyindependent index genetic variants $\left(\mathrm{p}<5 \times 10^{-8}\right)$. Individual-level genotypes for the conditionallyindependent variants identified in this first step were then extracted in 350,721 unrelated European ancestry participants of UK Biobank and their independent association was confirmed in multivariable linear regression models including all variants put forward from approximate analyses. Then, at each region, we statistically decomposed the identified index signals by conditioning on the other conditionally-independent index variants. We then performed Bayesian fine-mapping (62) to estimate the posterior probability of association for each variant (PPA, where $0 \%$ indicates that the variant is not causal and $100 \%$ indicates the highest possible posterior probability that the variant is causal) and define the $99 \%$ credible set at that signal (i.e. a set of variants in a genomic window that accounts for $99 \%$ of the PPA at that association signal). To generate credible sets, the association results at each locus were converted to Bayes factors (BF) for each variant within the locus boundary. The posterior probability that a variant- $\mathrm{j}$ was causal was defined by:

$$
\Phi_{j}=\frac{B F_{j}}{\sum_{k} B F_{k}}
$$

where, $\mathrm{BF}_{\mathrm{j}}$ denotes the $\mathrm{BF}$ for the $\mathrm{j}_{\mathrm{th}}$ variant, and the denominator is the sum of $\mathrm{BF}_{\mathrm{s}}$ for all included variants at that signal. A 99\% credible set of variants was created by ranking the posterior probabilities from highest to lowest and summing them until the cumulative posterior probability exceeded 0.99 (i.e. $99 \%$ ). 


\section{UK Biobank exome-sequence data processing and QC}

401 Whole exome sequencing (WES) data of 200,643 UK Biobank participants made available in October 2020

402 were downloaded in VCF and PLINK formats. The details of the UK Biobank WES data processing are 403 provided in detail elsewhere $(63,64)$. Further data processing and quality control has been described 404 previously (65). In brief, we did not apply additional QC based on QUAL (variant site-level quality score, 405 Phred scale) or AQ measures (variant site-level allele quality score reflecting evidence for each alternate 406 allele, Phred scale). Site-level filtering was applied for targeted biallelic calls if the AB ratio (no. of alternate 407 allele reads/total depth) was $\leq 0.25$ or $\geq 0.8$. Variant-level QC filters were applied if any of the variants had 408 (i) genotype missingness $>5 \%$, (ii) maximum read depth (DP) of less than 10 across samples or (iii) had GQ less than 20 for over $20 \%$ of the calls. After applying these filters, $7.3 \%$ of the variants were flagged as poor quality and not taken forward for further analysis.

\section{Variant annotation and definition of gene burden sets}

413 We annotated variants released in UK Biobank 200K whole exome sequencing VCF files in build hg38, using the Variant Effect Predictor (VEP) tool release 99 provided by Ensembl (66). In addition to default VEP features such as the consequence and impact of the variant, overlapping gene, position at cDNA and protein level and codon and amino acid change, if applicable, we have used the following plugins for annotation: (i) SIFT (67), which predicts whether an amino acid substitution affects protein function based on sequence homology and the physical properties of amino acid, (ii) Polyphen-2 (25), which predicts possible impact of an amino acid substitution on the structure and function of a human protein, (iii) CADD (27) which provides deleteriousness prediction scores for all variants based on diverse genomic features and (iv) LOFTEE (68)which provides loss of function prediction for variants. We annotated each variant using the most severe consequence across overlapping transcripts in Ensembl. We defined loss of function variants as those with 'high' impact predict by VEP. This includes frameshift variants, transcript ablating gained variants. 'Moderate impact' variants include missense variants, in-frame deletion or insertions, missense variants and protein altering variants.

\section{Gene-based association testing}


In our discovery stage, we used the method STAAR (variant-Set Test for Association using Annotation infoRmation), which is a computationally scalable method for very large whole-exome sequence (WES) and whole-exome sequence (WGS) studies and large-scale biobanks. STAAR uses a Generalized Linear Mixed Model (GLMM) framework that includes linear and logistic mixed models and can also account for both relatedness and population structure for both quantitative and dichotomous traits (69). In our analysis, we used the genotype dosage matrix as the genotype input and covariates including age at first check (age), age $^{2}$, sex, genotyping array, top ten genetically derived principal components (PC1-PC10) generated from the SNP array data, exome sequencing batch and the sparse GRM. For rank-based inverse normal transformed $\mathrm{WHR}_{\text {adjBMI}}$, we also added BMI as a covariate. We excluded the samples from our analysis if they did not pass UK Biobank quality control parameters, were non-European ancestry or if they withdrew consent from the study $(\mathrm{n}=184,246)(65)$.

We ran STAAR with its default options without additional functional annotations. For each gene, with at least two variants with $\mathrm{MAF} \leq 0.5 \%$, we conducted gene-based association analysis for the following three variant categories: rare variants predicted by VEP to be a) loss of function (pLoF; i.e. high impact), b) missense (Moderate; i.e. moderate impact) or c) both (pLoF+Moderate). For each variant clustering of a gene, STAAR will provide p-values for several collapsing burden tests including SKAT (sequence kernel association test), Burden test, and ACAT-V (set-based aggregated Cauchy association test). In addition, the output of STAAR also includes the omnibus p-value (STAAR-O) by using the combined Cauchy association test to aggregate the association across the different tests.

After identifying the genes with STAAR-O p-value over the threshold for exome-wide significance $\left(p<2.53 \times 10^{-6}\right)$, we applied more stringent QC filters on the genotype calls of the included variants. We set to missing genotype calls which did not meet the following QC criteria: 1. Genotype Quality (GQ) $\geq 20$ for heterozygous variants; 2 . Depth (DP) $\geq 7$ for SNVs and DP $\geq 10$ for InDels; 3. A binomial test on allelic balance using the Allelic Depth (AD) FORMAT field for heterozygous variants with $p \geq 1 \times 10^{-3}$. We then repeated the STAAR analysis using the filtered genotype dosage matrix.

To examine the extent to which the gene-based association is driven by single variants, we conducted a sensitivity leave-one-out analysis for each significant gene $\left(p<2.53 \times 10^{-6}\right)$, testing the significance of the gene-based association after excluding each variant.

\section{Secondary association testing}

We created dichotomous dummy variables using the filtered genotype dosage matrix for each identified gene, where samples with one or more rare alleles were set as " 1 " and the samples without rare alleles were 
set as " 0 " for different variant clustering settings of each gene. Then we combined these dummy variables into a single file and transformed it to BGEN format, which was used as the genotype input for association testing using a linear mixed model implemented in BOLT-LMM to account for cryptic population structure and relatedness (59). The GRM in BOLT-LMM was generated from the autosomal genetic variants that were common (MAF > 1\%), passed quality control in all 106 batches, and were present on both genotyping arrays (65). Covariates included age, sex, and PC1-PC10, genotyping chip and exome sequencing batch. For rank-based inverse normal transformed $\mathrm{WHR}_{\text {adjBMI, }}$ covariates also included BMI. We excluded the same group of samples as we did for STAAR analyses.

To test for heterogeneity of effect sizes between men and women for significant genes identified in the gene-based analyses, we used a Z-test to compare effect size estimates for each gene calculated in the sexspecific analyses.

\section{Phenotypic associations}

The gene-based phenotypic associations using the same STAAR and BOLT-LMM pipelines for the following continuous phenotypes: BMI, BIA-derived gynoid fat, BIA-derived leg fat, BIA-derived android fat, BIA-derived trunk fat, BIA-derived arm fat, triglyceride levels, HDL cholesterol, LDL cholesterol, HbA1c levels (see Supplementary Table 12 for phenotype details). Body fat compartments were predicted using bioimpedance measurements in UK Biobank. The details for the prediction of body fat compartments in UK Biobank are described elsewhere (16).

We have also investigated gene-based phenotypic associations for binary disease outcomes: type 2 diabetes and cardiovascular heart disease. As BOLT-LMM is based on the linear mixed model which cannot give an accurate effect estimate for binary variables, we have also applied a generalized linear model (GLM) to estimate the Odd Ratio (OR) for binary phenotypes. We also looked up these binary outcomes in other resources such as the AstraZeneca PheWAS Portal (https://azphewas.com/, accessed on 02/09/2021) (14) and the Type 2 Diabetes Knowledge Portal (T2DKP; https://t2d.hugeamp.org/, accessed on 02/09/2021). The AstraZeneca PheWAS Portal also uses UK Biobank as their primary resource, but have access to a larger dataset of 281,104 exomes. We looked up results for T2D ( $\mathrm{N}$ cases $=1,671 ; \mathrm{N}$ controls $=160,949)$ and chronic ischaemic heart disease (defined by ICD-10 code I25; $\mathrm{N}$ cases $=24,147$, $\mathrm{N}$ controls $=176,170$ ). In the T2DKP, we also looked up results for T2D $(\mathrm{N}=43,125)$. 


\section{ACVR1C dual-luciferase assay}

HEK293 cells were seeded at a density of 150,000 cells per well in 24-well tissue culture plates pre-treated with poly-D-lysine. On the following day, medium was replaced with Opti-MEM I Reduced Serum medium and a total of $550 \mathrm{ng}$ of plasmid DNA; this included different pcDNA3.1 based ACVR1C constructs listed in the table below, as well as constructs encoding receptor components (ACVR-IIB and CRIPTO) along with firefly (consisting of the SMAD binding elements) and Renilla (control) luciferase reporter plasmids. Lipofectamine 3000 Reagent was used for the transfection according to the manufacturer's protocol. OptiMEM I Reduced Serum medium was then replaced with DMEM growth medium 6 hours post transfection.

\begin{tabular}{|l|l|}
\hline pcDNA3.1 construct & \multicolumn{1}{|c|}{ Receptor components } \\
\hline WT & ACVR-IIB, CRIPTO, pGL4.48[luc2P/SBE/Hygro], pRL-SV40 \\
\hline I195T & ACVR-IIB, CRIPTO, pGL4.48[luc2P/SBE/Hygro], pRL-SV40 \\
\hline K222R & ACVR-IIB, CRIPTO, pGL4.48[luc2P/SBE/Hygro], pRL-SV40 \\
\hline T194D & ACVR-IIB, CRIPTO, pGL4.48[luc2P/SBE/Hygro], pRL-SV40 \\
\hline EV (empty vector) & ACVR-IIB, CRIPTO, pGL4.48[luc2P/SBE/Hygro], pRL-SV40 \\
\hline
\end{tabular}

The dual-luciferase reporter assay was performed according to the manufacturer's protocol (Promega, USA). Cells were washed once with DPBS, followed by an active lysis procedure. Briefly, $125 \mu$ l of passive lysis buffer was added in each well and the cells were subjected to one cycle of a freeze-thaw process. Cell lysates were cleared of cell debris by centrifugation at 21,130 $\mathrm{g}$ for one minute. The assay was conducted in a 96-well plate format. In each assay, $20 \mu \mathrm{l}$ of cleared supernatant was pre-dispensed, followed by sequential measurement of firefly and Renilla luciferase using a Tecan Spark 10M plate reader (Tecan, Switzerland). Firefly luciferase activity was normalised for Renilla luciferase activity, and then further normalised with values from non-stimulated cells transfected with empty pcDNA3.1 vector (EV). We also studied a constitutively active (ACVR1C p.T194D) mutant and a kinase dead (ACVR1C p.K222R) mutant for comparison $(70,71)$. The experiment was repeated with fresh transfections on three separate occasions. 
516

517

518

519

520

521

522

523

524

525

526

527

528

529

530

531

532

533

534

535

536

537

538

539

540

541

542

543

544

545

546

547

\section{REFERENCES}

1. Lotta LA, Wittemans LBL, Zuber V, Stewart ID, Sharp SJ, Luan J, et al. Association of Genetic Variants Related to Gluteofemoral vs Abdominal Fat Distribution With Type 2 Diabetes, Coronary Disease, and Cardiovascular Risk Factors. JAMA. 2018;320(24):2553-63.

2. Shungin D, Winkler TW, Croteau-Chonka DC, Ferreira T, Locke AE, Mägi R, et al. New genetic loci link adipose and insulin biology to body fat distribution. Nature. 2015;518(7538):187-96.

3. Pulit SL, Stoneman C, Morris AP, Wood AR, Glastonbury CA, Tyrrell J, et al. Meta-analysis of genome-wide association studies for body fat distribution in 694649 individuals of European ancestry. Hum Mol Genet. 2019;28(1):166-74.

4. Abecasis GR, Auton A, Brooks LD, DePristo MA, Durbin RM, Handsaker RE, et al. An integrated map of genetic variation from 1,092 human genomes. Nature. 2012;491(7422):56-65.

5. Claussnitzer M, Cho JH, Collins R, Cox NJ, Dermitzakis ET, Hurles ME, et al. A brief history of human disease genetics. Nature. 2020;577(7789):179-89.

6. Wang Q, Dhindsa RS, Carss K, Harper A, Nag A, Tachmazidou I, et al. Surveying the contribution of rare variants to the genetic architecture of human disease through exome sequencing of 177,882 UK Biobank participants. bioRxiv. 2020:2020.12.13.422582.

7. Raychaudhuri S. Mapping rare and common causal alleles for complex human diseases. Cell. 2011;147(1):57-69.

8. McCarthy S, Das S, Kretzschmar W, Delaneau O, Wood AR, Teumer A, et al. A reference panel of 64,976 haplotypes for genotype imputation. Nat Genet. 2016;48(10):1279-83.

9. Justice AE, Karaderi T, Highland HM, Young KL, Graff M, Lu Y, et al. Protein-coding variants implicate novel genes related to lipid homeostasis contributing to body-fat distribution. Nat Genet. 2019;51(3):452-69.

10. Akbari P, Gilani A, Sosina O, Kosmicki JA, Khrimian L, Fang YY, et al. Sequencing of 640,000 exomes identifies GPR75 variants associated with protection from obesity. Science. 2021;373(6550).

11. Dewey FE, Murray MF, Overton JD, Habegger L, Leader JB, Fetterolf SN, et al. Distribution and clinical impact of functional variants in 50,726 whole-exome sequences from the DiscovEHR study. Science. 2016;354(6319).

12. Van Hout CV, Tachmazidou I, Backman JD, Hoffman JD, Liu D, Pandey AK, et al. Exome sequencing and characterization of 49,960 individuals in the UK Biobank. Nature. 2020;586(7831):749-56.

13. Park J, Lucas AM, Zhang X, Chaudhary K, Cho JH, Nadkarni G, et al. Exome-wide evaluation of rare coding variants using electronic health records identifies new gene-phenotype associations. Nat Med. 2021;27(1):66-72. 
14. Wang Q, Dhindsa RS, Carss K, Harper AR, Nag A, Tachmazidou I, et al. Rare variant contribution to human disease in 281,104 UK Biobank exomes. Nature. 2021.

15. Granneman JG, Moore HP, Krishnamoorthy R, Rathod M. Perilipin controls lipolysis by regulating the interactions of AB-hydrolase containing 5 (Abhd5) and adipose triglyceride lipase (Atgl). J Biol Chem. 2009;284(50):34538-44.

16. Powell R, De Lucia Rolfe E, Day FR, Perry JRB, Griffin SJ, Forouhi NG, et al. Development and validation of total and regional body composition prediction equations from anthropometry and single frequency segmental bioelectrical impedance with DEXA. medRxiv. 2020:2020.12.16.20248330.

17. McLaughlin T, Abbasi F, Cheal K, Chu J, Lamendola C, Reaven G. Use of metabolic markers to identify overweight individuals who are insulin resistant. Ann Intern Med. 2003;139(10):802-9.

18. Freeman AM, Pennings N. Insulin Resistance. StatPearls. Treasure Island (FL): StatPearls Publishing

Copyright (C) 2021, StatPearls Publishing LLC.; 2021.

19. Zhu Z, Guo Y, Shi H, Liu CL, Panganiban RA, Chung W, et al. Shared genetic and experimental links between obesity-related traits and asthma subtypes in UK Biobank. J Allergy Clin Immunol. 2020;145(2):537-49.

20. Semple RK, Savage DB, Cochran EK, Gorden P, O'Rahilly S. Genetic syndromes of severe insulin resistance. Endocr Rev. 2011;32(4):498-514.

21. Emdin CA, Khera AV, Aragam K, Haas M, Chaffin M, Klarin D, et al. DNA Sequence Variation in ACVRIC Encoding the Activin Receptor-Like Kinase 7 Influences Body Fat Distribution and Protects Against Type 2 Diabetes. Diabetes. 2019;68(1):226-34.

22. Jagadeesh KA, Wenger AM, Berger MJ, Guturu H, Stenson PD, Cooper DN, et al. M-CAP eliminates a majority of variants of uncertain significance in clinical exomes at high sensitivity. Nat Genet. 2016;48(12):1581-6.

23. Ioannidis NM, Rothstein JH, Pejaver V, Middha S, McDonnell SK, Baheti S, et al. REVEL: An Ensemble Method for Predicting the Pathogenicity of Rare Missense Variants. Am J Hum Genet. 2016;99(4):877-85.

24. Ng PC, Henikoff S. SIFT: Predicting amino acid changes that affect protein function. Nucleic Acids Res. 2003;31(13):3812-4.

25. Adzhubei I, Jordan DM, Sunyaev SR. Predicting functional effect of human missense mutations using PolyPhen-2. Curr Protoc Hum Genet. 2013; Chapter 7:Unit7.20.

26. Choi Y, Chan AP. PROVEAN web server: a tool to predict the functional effect of amino acid substitutions and indels. Bioinformatics. 2015;31(16):2745-7. 
27. Rentzsch P, Witten D, Cooper GM, Shendure J, Kircher M. CADD: predicting the deleteriousness of variants throughout the human genome. Nucleic Acids Res. 2019;47(D1):D886-D94.

28. Sinnott-Armstrong N, Tanigawa Y, Amar D, Mars N, Benner C, Aguirre M, et al. Genetics of 35 blood and urine biomarkers in the UK Biobank. Nat Genet. 2021;53(2):185-94.

29. Klarin D, Damrauer SM, Cho K, Sun YV, Teslovich TM, Honerlaw J, et al. Genetics of blood lipids among $~ 300,000$ multi-ethnic participants of the Million Veteran Program. Nat Genet. 2018;50(11):1514-23.

30. Vuckovic D, Bao EL, Akbari P, Lareau CA, Mousas A, Jiang T, et al. The Polygenic and Monogenic Basis of Blood Traits and Diseases. Cell. 2020;182(5):1214-31.e11.

31. Emdin CA, Khera AV, Chaffin M, Klarin D, Natarajan P, Aragam K, et al. Analysis of predicted loss-of-function variants in UK Biobank identifies variants protective for disease. Nat Commun. 2018;9(1):1613.

32. Pischon T, Boeing H, Hoffmann K, Bergmann M, Schulze MB, Overvad K, et al. General and abdominal adiposity and risk of death in Europe. N Engl J Med. 2008;359(20):2105-20.

33. Wang Y, Rimm EB, Stampfer MJ, Willett WC, Hu FB. Comparison of abdominal adiposity and overall obesity in predicting risk of type 2 diabetes among men. Am J Clin Nutr. 2005;81(3):555-63.

34. Canoy D. Distribution of body fat and risk of coronary heart disease in men and women. Curr Opin Cardiol. 2008;23(6):591-8.

35. Mason C, Craig CL, Katzmarzyk PT. Influence of central and extremity circumferences on allcause mortality in men and women. Obesity (Silver Spring). 2008;16(12):2690-5.

36. Bogardus C, Lillioja S, Mott DM, Hollenbeck C, Reaven G. Relationship between degree of obesity and in vivo insulin action in man. Am J Physiol. 1985;248(3 Pt 1):E286-91.

37. Hocking S, Samocha-Bonet D, Milner KL, Greenfield JR, Chisholm DJ. Adiposity and insulin resistance in humans: the role of the different tissue and cellular lipid depots. Endocr Rev. 2013;34(4):463500.

38. Rose KM, Newman B, Mayer-Davis EJ, Selby JV. Genetic and behavioral determinants of waisthip ratio and waist circumference in women twins. Obes Res. 1998;6(6):383-92.

39. Lim K, Haider A, Adams C, Sleigh A, Savage DB. Lipodistrophy: a paradigm for understanding the consequences of "overloading" adipose tissue. Physiol Rev. 2021;101(3):907-93.

40. Emdin CA, Khera AV, Natarajan P, Klarin D, Zekavat SM, Hsiao AJ, et al. Genetic Association of Waist-to-Hip Ratio With Cardiometabolic Traits, Type 2 Diabetes, and Coronary Heart Disease. JAMA. 2017;317(6):626-34.

41. Locke AE, Kahali B, Berndt SI, Justice AE, Pers TH, Day FR, et al. Genetic studies of body mass index yield new insights for obesity biology. Nature. 2015;518(7538):197-206. 
42. Fischer JP, Els-Heindl S, Beck-Sickinger AG. Adrenomedullin - Current perspective on a peptide hormone with significant therapeutic potential. Peptides. 2020;131:170347.

43. Erratum to: "Altered Expression of Adrenomedullin 2 and its Receptor in the Adipose Tissue of Obese Patients". J Clin Endocrinol Metab. 2020;105(12).

44. Sztalryd C, Brasaemle DL. The perilipin family of lipid droplet proteins: Gatekeepers of intracellular lipolysis. Biochim Biophys Acta Mol Cell Biol Lipids. 2017;1862(10 Pt B):1221-32.

45. Degerman E, Ahmad F, Chung YW, Guirguis E, Omar B, Stenson L, et al. From PDE3B to the regulation of energy homeostasis. Curr Opin Pharmacol. 2011;11(6):676-82.

46. DiPilato LM, Ahmad F, Harms M, Seale P, Manganiello V, Birnbaum MJ. The Role of PDE3B Phosphorylation in the Inhibition of Lipolysis by Insulin. Mol Cell Biol. 2015;35(16):2752-60.

47. Yogosawa S, Mizutani S, Ogawa Y, Izumi T. Activin receptor-like kinase 7 suppresses lipolysis to accumulate fat in obesity through downregulation of peroxisome proliferator-activated receptor $\gamma$ and C/EBP $\alpha$. Diabetes. 2013;62(1):115-23.

48. Ibáñez CF. Regulation of metabolic homeostasis by the TGF- $\beta$ superfamily receptor ALK7. FEBS J. 2021.

49. Guo T, Marmol P, Moliner A, Björnholm M, Zhang C, Shokat KM, et al. Adipocyte ALK7 links nutrient overload to catecholamine resistance in obesity. Elife. 2014;3:e03245.

50. Laver TW, Patel KA, Colclough K, Curran J, Dale J, Davis N, et al. PLIN1 Haploinsufficiency Is Not Associated With Lipodystrophy. J Clin Endocrinol Metab. 2018;103(9):3225-30.

51. Jéru I, Vantyghem MC, Bismuth E, Cervera P, Barraud S, Auclair M, et al. Diagnostic Challenge in PLIN1-Associated Familial Partial Lipodystrophy. J Clin Endocrinol Metab. 2019;104(12):6025-32.

52. Gandotra S, Le Dour C, Bottomley W, Cervera P, Giral P, Reznik Y, et al. Perilipin deficiency and autosomal dominant partial lipodystrophy. N Engl J Med. 2011;364(8):740-8.

53. Chen W, Chang B, Wu X, Li L, Sleeman M, Chan L. Inactivation of Plin4 downregulates Plin5 and reduces cardiac lipid accumulation in mice. Am J Physiol Endocrinol Metab. 2013;304(7):E770-9.

54. Lagou V, Mägi R, Hottenga JJ, Grallert H, Perry JRB, Bouatia-Naji N, et al. Sex-dimorphic genetic effects and novel loci for fasting glucose and insulin variability. Nat Commun. 2021;12(1):24.

55. Garg A. Gender differences in the prevalence of metabolic complications in familial partial lipodystrophy (Dunnigan variety). J Clin Endocrinol Metab. 2000;85(5):1776-82.

56. Mann JP, Savage DB. What lipodystrophies teach us about the metabolic syndrome. J Clin Invest. 2019;129(10):4009-21.

57. Bycroft C, Freeman C, Petkova D, Band G, Elliott LT, Sharp K, et al. The UK Biobank resource with deep phenotyping and genomic data. Nature. 2018;562(7726):203-9. 
58. Wang K, Li M, Hakonarson H. ANNOVAR: functional annotation of genetic variants from highthroughput sequencing data. Nucleic Acids Res. 2010;38(16):e164.

59. Loh PR, Tucker G, Bulik-Sullivan BK, Vilhjálmsson BJ, Finucane HK, Salem RM, et al. Efficient Bayesian mixed-model analysis increases association power in large cohorts. Nat Genet. 2015;47(3):28490.

60. Yang J, Lee SH, Goddard ME, Visscher PM. GCTA: a tool for genome-wide complex trait analysis. Am J Hum Genet. 2011;88(1):76-82.

61. Yang J, Ferreira T, Morris AP, Medland SE, Madden PA, Heath AC, et al. Conditional and joint multiple-SNP analysis of GWAS summary statistics identifies additional variants influencing complex traits. Nat Genet. 2012;44(4):369-75, S1-3.

62. Maller JB, McVean G, Byrnes J, Vukcevic D, Palin K, Su Z, et al. Bayesian refinement of association signals for 14 loci in 3 common diseases. Nat Genet. 2012;44(12):1294-301.

63. Szustakowski JD, Balasubramanian S, Kvikstad E, Khalid S, Bronson PG, Sasson A, et al. Advancing human genetics research and drug discovery through exome sequencing of the UK Biobank. Nat Genet. 2021;53(7):942-8.

64. Yun T, Li H, Chang PC, Lin MF, Carroll A, McLean CY. Accurate, scalable cohort variant calls using DeepVariant and GLnexus. Bioinformatics. 2021.

65. Zhao Y, Stankovic S, Koprulu M, Wheeler E, Day FR, Lango Allen H, et al. GIGYF1 loss of function is associated with clonal mosaicism and adverse metabolic health. Nat Commun. 2021;12(1):4178. 66. McLaren W, Gil L, Hunt SE, Riat HS, Ritchie GR, Thormann A, et al. The Ensembl Variant Effect Predictor. Genome Biol. 2016;17(1):122.

67. Kumar P, Henikoff S, Ng PC. Predicting the effects of coding non-synonymous variants on protein function using the SIFT algorithm. Nat Protoc. 2009;4(7):1073-81.

68. Karczewski K. Loftee (Loss-of-Function Transcript Effect Estimator) 2015.

69. Li X, Li Z, Zhou H, Gaynor SM, Liu Y, Chen H, et al. Dynamic incorporation of multiple in silico functional annotations empowers rare variant association analysis of large whole-genome sequencing studies at scale. Nat Genet. 2020;52(9):969-83.

70. Bondestam J, Huotari MA, Morén A, Ustinov J, Kaivo-Oja N, Kallio J, et al. cDNA cloning, expression studies and chromosome mapping of human type I serine/threonine kinase receptor ALK7 (ACVR1C). Cytogenet Cell Genet. 2001;95(3-4):157-62.

71. Wieser R, Wrana JL, Massagué J. GS domain mutations that constitutively activate T beta R-I, the downstream signaling component in the TGF-beta receptor complex. EMBO J. 1995;14(10):2199-208. 


\section{Acknowledgements}

This research has been conducted using the UK Biobank resource. Access to the UK Biobank genotype and phenotype data is open to all approved health researchers (http://www.ukbiobank.ac.uk/). This study was funded by the United Kingdom's Medical Research Council through grants MC_UU_12015/1, MC_PC_13046, MC_PC_13048 and MR/L00002/1. This work was supported by the MRC Metabolic Diseases Unit (MC_UU_12012/5) and the Cambridge NIHR Biomedical Research Centre and EU/EFPIA Innovative Medicines Initiative Joint Undertaking (EMIF grant: 115372). R.K.S, D.B.S. and S.O'R. are supported by the Wellcome Trust (WT 210752, WT 219417 and WT 214274 respectively) the MRC Metabolic Disease Unit, the National Institute for Health Research (NIHR) Cambridge Biomedical Research Centre and the NIHR Rare Disease Translational Research Collaboration. K.S.S. is supported by MRC Project Grant L01999X/1. Some computation was enabled through access granted to K.S.S. to the MRC eMedLab Medical Bioinformatics infrastructure, supported by the Medical Research Council (grant number MR/L016311/1). M.McC. is a Wellcome Senior Investigator supported by Wellcome grants 098381, 090532, 106130, 203141. M.McC. declares that the views expressed in this article are those of the authors and not necessarily those of the NHS, the NIHR, or the Department of Health; he has served on advisory panels for Pfizer, Novo Nordisk and Zoe Global, has received honoraria from Merck, Pfizer, Novo Nordisk and Eli Lilly, and research funding from Abbvie, Astra Zeneca, Boehringer Ingelheim, Eli Lilly, Janssen, Merck, Novo Nordisk, Pfizer, Roche, Sanofi Aventis, Servier, and Takeda. M.K. is supported by the Gates Cambridge Trust. The authors gratefully acknowledge the help of the MRC Epidemiology Unit Support Teams, including Field, Laboratory and Data Management Teams.

\section{Competing interests}

M.McM. is an employee of Genentech, and a holder of Roche stock. D.M.E.L. is currently an employee of Enhanc3D Genomics Ltd. N.B. is an employee of GlaxoSmithKline Plc. (GSK). R.A.S. is an employee and shareholder of GlaxoSmithKline Plc. (GSK). C.A.G. is an employee of Benevolent AI. L.A.L. is an employee of Regeneron Genetics Center and receives salary, stocks and stock options from Regeneron Pharmaceuticals Inc.

\section{Author contributions}

Data collection and analysis: M.K., Y.Z., E.W., L.D., N.R., S.P., M.V.S., C.G., I.D.S, F.R.D, J.L., N.B., L.B.L.W., N.D.K., V.S.,

Study supervision: D.M.E.L., I.B., M.I.McM., R.A.S., K.S.S., N.J.W., R.K.S., L.A.L, J.R.B.P., S.O'R., C.L., D.B.S. 
medRxiv preprint doi: https://doi.org/10.1101/2021.09.11.21263427; this version posted September 15, 2021. The copyright holder for this preprint (which was not certified by peer review) is the author/funder, who has granted medRxiv a license to display the preprint in perpetuity.

It is made available under a CC-BY-NC-ND 4.0 International license .

716

717 Data availability

718 This research has been conducted using the UK Biobank resource (application no. 44448 and 9905).

719 Access to the UK Biobank genotype and phenotype data is open to all approved health researchers

720 (http://www.ukbiobank.ac.uk/). 